\title{
Intelligent Agents for a Mobile Network Manager (MNM)
}

\author{
Akhil Sahai ${ }^{\dagger}$, Christine Morin ${ }^{\dagger}$, Stéphane Billiart ${ }^{\ddagger}$ \\ $\dagger$ INRIA \\ $\ddagger B U L L$ \\ IRISA, Campus Universitaire de Beaulieu \\ 35042 Rennes Cedex (France) \\ \{asahai, cmorin, billiart\}@irisa.fr
}

\begin{abstract}
MAGENTA (Mobile AGENT for Administration) is our mobile agent environment for network management. The MAGENTA environment is being developed under the action Astrolog* which is a highly dynamic and decentralized management system meant for the management of Astrolab, a distributed system comprising of heterogeneous machines running varied operating systems connected by a LAN. The MAGENTA environment enables the introduction of a unique idea of a Mobile Network Manager(MNM).
\end{abstract}

Keywords

Mobile Agents, Mobile Computing, Network Management, SNMP, Java

\section{INTRODUCTION}

The research project Astrolog intends to design a management environment for the Astrolab environment. The Astrolab environment comprises of a variety of machines running different Operating systems like PCs (executing Win3.1, Win95, WinNT, Linux, NetBSD) and many variants of POSIX environment. Astrolog introduces the concept of a simple, portable, light-weight and cost-effective manager for management of our local network. The manager being light-weight and portable can be executed from either a static computer or a mobile computer. The manager operating from a mobile computer is termed a Mobile Network Manager (MNM).

Under the action astrolog we are implementing the MAGENTA (Mobile AGENT for Administration) environment to enable the functioning of the

*Part of this work is being carried out under the GIE-DYADE collaboration between INRIA and BULL. 
MNM and to decentralize network management functions. The MAGENTA environment is highly generic in nature and can be utilized for the administration of any kind of services including information retrieval, electronic commerce and data mining.

In this paper, we present the network management architecture of Astrolog and describe the design and implementation of the MNM. We also present the MAGENTA environment and also describe, how it is utilized to enable the functioning of the MNM. The rest of the paper is structured as follows. The subsequent section provides an overview of the mobile agents. This, is followed by an overview of network management systems.Section 4 presents an overview of the Astrolog architecture. Section 5 presents the MAGENTA environment. This is followed by the implementation details before concluding.

\section{OVERVIEW OF INTELLIGENT MOBILE AGENTS}

In lieu of the client-server paradigm which has not been able to cope with the various kinds of demands put on it, the mobile agent based computing approach has been propounded as the possible approach to the next generation computing (Colin et al. 1995). With the rapid proliferation of available data and its sources the users have been increasingly finding it difficult to obtain, analyze and utilize the available data in a coherent manner. the agent based computing has thus been proposed as a solution to lessen the burden of the users. The agent based computing has also been shown to be more effective in the case of partially connected computing.

In the weaker notion of agency an agent has been variously described as an autonomous program acting on behalf of the user and working under constraints predetermined by the user in order to accomplish a task or as an assistant of a user delegated to perform activities autonomously in order to lessen his responsibilities. Of late, the term agent has been increasingly used and hence it lacks a coherent description. An effort has been made in (Jennings, Wooldridge 1994) to study the hallmarks of agent-hood. The hallmarks have been put down namely as autonomy, social ability, responsiveness and proactiveness. The agents available are numerous and differ substantially in their functionality and performance. In (Beale et al. 1994), an effort to classify the various types of available agents has been made. The various types of agents have been classified as user agents, agent guides, autonomous agents, symbiotic and cooperative agents, anthropomorphic agents, agents for multi-agent systems, and agents for agent-oriented programming.

In the stronger notion of agency the agents along with the above mentioned capabilities are intended to possess human like qualities and behaviour, specially in the field of AI. The agents are endowed with notions like knowl- 
edge, belief, intention and obligation (Reilly, Bates 1995). The agents in some cases have mental states and sometimes are given human like visualization (Maes 1994).

Mobile agents have been proposed for various applications like electronic commerce, desktop applications, information retrieval, data-mining, messaging, user interface and network management. In the case of network management, they have been proposed for Telecommunication management Network (TMN) as in (Magedanz et al. 1996) and as generalized scripted and delegated agents for management in (Goldzmith, Yemini 1995). In (Goldzmith, Yemini 1995) the mobile agents are sent to remote sites where they are incorporated into the local network management program and are used for intelligent tasks like MIB (Management Information Base) filtering. These are mostly one hop agents and they are used to avoid transfer of data over the network and to execute management logic close to the remote site. However, these agents are not generic and are not portable. In (Krause 1997) the mobile agents are used to ameliorate the service scalability problem inherent in Intelligent Networks. They intend to utilize the mobile agent technology to provide telecommunication services instantly and to be customized directly at the locations where the intelligence is needed. CyberAgents (CyberAgent. 1997) are being sold as a commercial product and are intended for simple network management configuration applications.

Some agent implementations have been proposed and carried out (Gray 1995) (Johansen et al. 1995) (Telescript. 1996). The mobile agents we utilize are however generalized and highly portable in nature and are attuned particularly to the needs of working in a constrained and fallible environment thus enabling the development of an MNM. In MAGENTA environment mobile agents are programs that can move through a network under their own control migrating from host to host and interacting with other agents and resources on each of the hosts. This is thus an extension to the client/server model in which the client and server exchange messages during execution. Mobile agents are an effective paradigm for distributed computing and are particularly attractive for partially connected computing.

\section{OVERVIEW OF NETWORK MANAGEMENT SYSTEMS}

Network management systems essentially have the functionality of network monitoring and network control. Network monitoring is concerned with monitoring the state of the network without interference. While network control involves active participation and interference in the state of the network.

The network-monitoring portion of network-management is concerned with observing and analyzing the status of the network to be managed. Network 
monitoring is an essential aspect of automated network management. The information to be gathered includes static information, related to the configuration, dynamic information related to events in the network and statistical information, summarized from dynamic information. It also involves identification of faults and determining the reasons of the fault and taking remedial actions. It also involves proactive response to an impending fault and minimization and containment of the fault.

Network control is concerned with changing the variable values of various components of the network and causing those components to perform predefined actions. The area of configuration control encompasses a variety of functions relating to the configuration of network and computing elements. These include initialization, maintenance, and shutdown of individual components and logical subsystems. In the area of security control, the responsibility of the network management system is to coordinate and control the security mechanisms built into the configuration of networks and systems under its management control. These security mechanisms are intended to protect user and system resources, including the network management system itself.

A network management system contains four types of components: Network Management Stations (NMSs), agents running on managed nodes, management protocols, and management information. An NMS uses the management protocol to communicate with agents running on the managed nodes. The information communicated between the NMS and agents is defined by a Management Information Base (MIB). The management standards that have emerged are the Simple Network Management Protocol (SNMP) and the OSI management system which utilizes the Common Management Information Protocol (CMIP)(Warrier, Besaw. 1989). SNMP is simpler and more concise in comparison to CMIP which is more elaborate and provides many more functionalities.

Astrolog utilizes SNMP in keeping with its intention of being light-weight and simple. The SNMP protocol includes the following capabilities mainly,

- Get: enables the management station to retrieve the value of objects from the agent;

- Set: enables the management station to set the value of objects at the agent;

- Trap: enables an agent to notify the management station of significant events.

The prevalent architectures of network management systems are

- Centralized network management. A single centralized manager overlooks the management. It queries the network components on a timely basis to 
determine the health of the network. In this case, there is a centralized database located at the site of the manager which stores and provides the information about the network components.

- Hierarchical network management. A central manager is aided by a set of subordinate managers. The subordinate managers take off some of the responsibilities of the central manager. The central manager performs the role of overall manager and has the centralized database. The subordinate managers in turn manage their domains.

- Peer network management. A set of network managers manage the different domains of the network with timely interaction amongst them. Each of the peer managers have a database and thus the information stored is partitioned and to an extent replicated.

- Fully distributed network management. A totally distributed management architecture in which every agent shares the responsibility of management. The managers have information which pertains to their domain and thus the overall information is highly partitioned.

\section{ASTROLOG OVERVIEW}

Astrolog (Sahai et al. 1997(1))(Sahai et al. 1997(2)) is our management platform for managing our local research platform called Astrolab which comprises of heterogeneous machines running several different operating systems connected by LAN. Thus, it was necessary for us to have a light-weight, portable and cost-effective manager. The idea was not only to make a light and simple manager for our local platform but also to introduce new ideas in the realm of network management.

The design of Astrolog is highly modular in nature. This modularity enables the development of a light-weight manager which can be executed from a mobile computer. In Astrolog, there is a provision of multiple managers which are designed to be portable and can run from a variety of machines over the network in a client-server mode or in the mobile agent mode or sometimes utilizing both. These managers communicate with one or multiple servers (depending on the size of the network) running on sites containing databases. The databases are populated by respective discovery daemons which gather information about the network components and store them in the database. These values are updated timely by obtaining SNMP values from the SNMP agents running on the managed machines. Another daemon obtains the required information from the database or directly from the agents located at the managed system components, as and when requested by the managers. As compared to a typical centralized management system in which the centralized manager comprises of the GUI, the management applications 


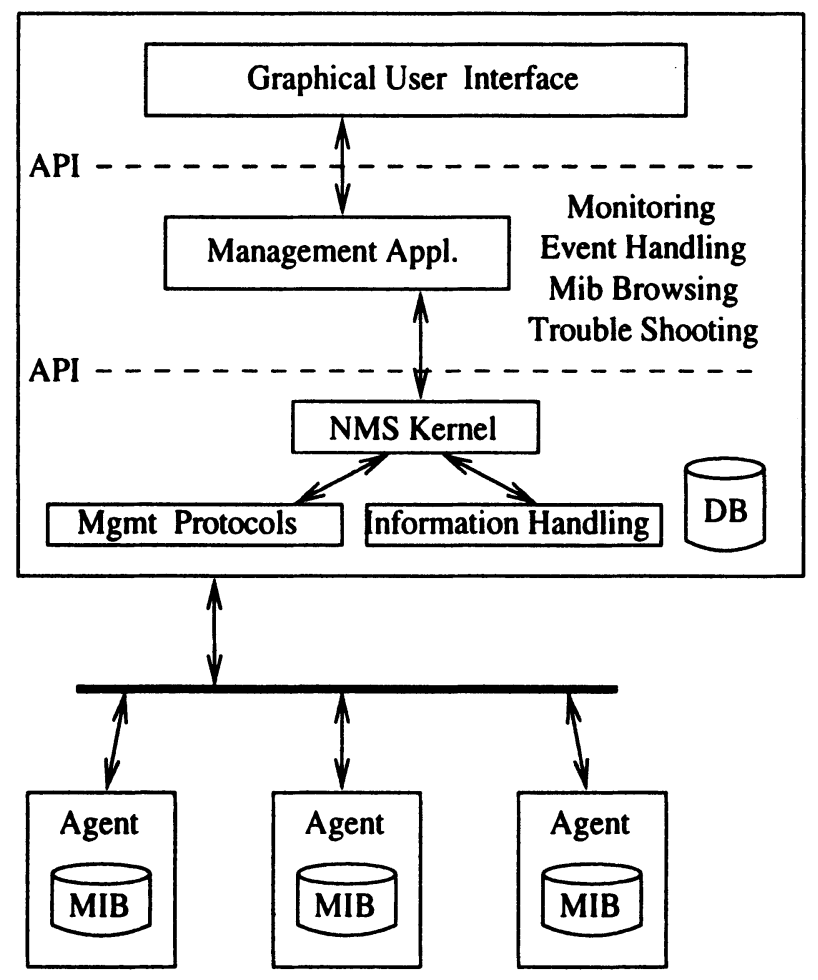

Figure 1 A typical centralized Network Management System

and the NMS kernel along with the database as shown in Figure 1, Astrolog is divided into light-weight managers comprising of the GUI and the management applications like MIB browsing and monitoring and a mechanism for connecting to the server and obtaining the required management information. The other part comprises of the NMS kernel (code which acts as the counterpart of the applications on the manager side and daemons), the database (DB) and a communication mechanism to interact with the managers, which in turn acts as the management server. There can be multiple managers querying one or more management servers. The managers can either exist on the same site as the server or can also exist at a different site and can query the server over the network as shown in Figure 2.

What facilitates the flexibility of the architecture is that the network managers are light-weight and thus there can be multiple interchangeable managers. We are utilizing this light-weightedness of the manager to build MNMs. Now-a-days in the event of a crisis the system administrator is informed by the management system through a pager message. One of the recourse left to the administrator is to rush to the central management station. In case of the absence of the system administrator in the proximity of the centralized 


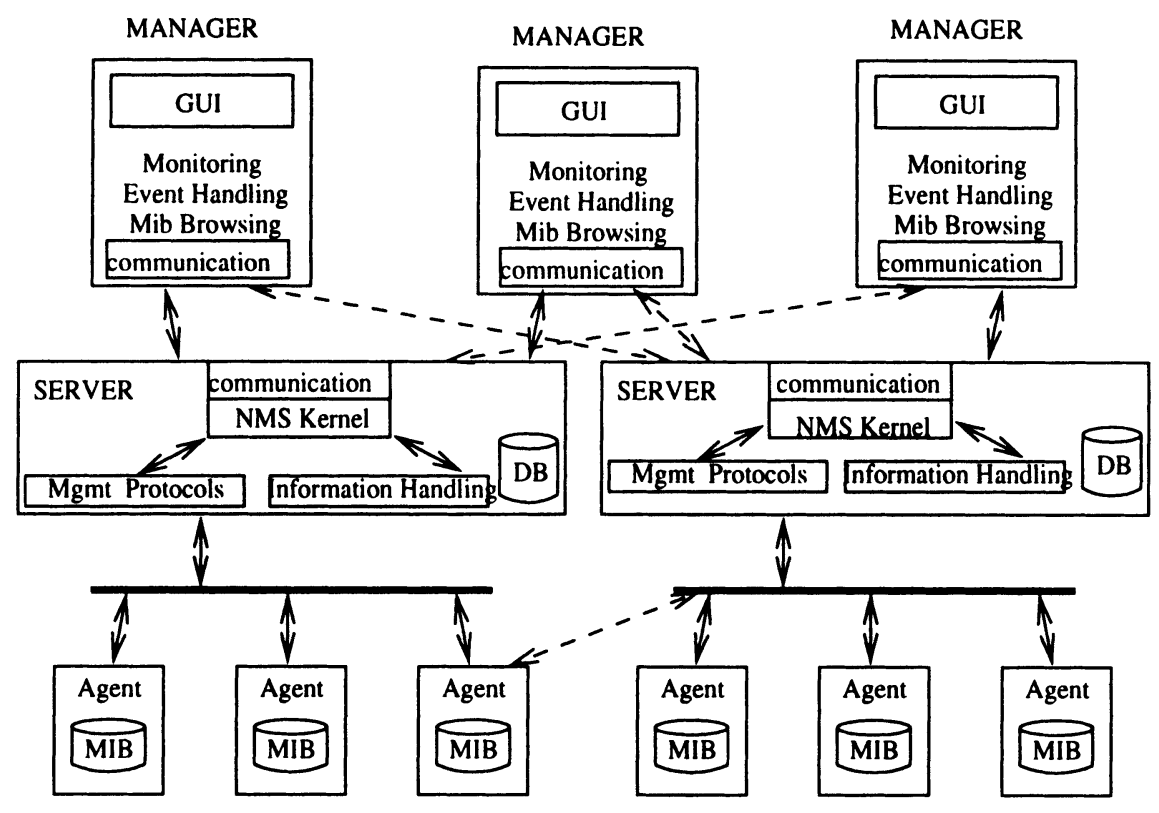

Figure 2 Astrolog Network Management System

management station, the system administrator is unaware of exact diagnostic information about the cause of the crisis and is thus extremely difficult for the administrator to undertake important decisions in case of a network breakdown. In case of a large network, there can be more than one administrators and it is sometimes essential to seek the opinion of more than one administrators at the same time. Also there is an increasing trend towards wireless networks and which according to us require a wireless network manager, thus we consider that there is an ample need and usefulness of MNMs . MNMs we propose are managers that run on portable computers which can either run in the tethered mode using a PPP/SLIP mechanism or can run on a roaming wireless computer (Gray et al. 1996). Our design takes care of both the situations.

What distinguishes these mobile computers are the extreme constraints on the link available to them. The links have serious bandwidth constraints, have high latency and are prone to sudden failures such as when a signal from a cellular modem is blocked by an obstacle. The computer may be forced to use different transmission channels depending on its physical location. Finally depending on the nature of the transmission channel, the computer may be assigned a different network address each time it connects. Both wireless networks and phone lines are orders of magnitude more constrained than traditional LANs (Oracle. 1995) as shown in Table 1 and in Table 2. Table 1 
Table 1 Comparison of Networks (from (Oracle. 1995))

\begin{tabular}{lrr}
\hline Networks & $B W($ Kbps) & Latency (secs) \\
\hline Wireless WAN & $2 \mathrm{~K}-9$ & $4-10$ \\
Modem & $2.4 \mathrm{~K}-28.8$ & $0.2-0.5$ \\
LAN & $5 \mathrm{~K}-10 \mathrm{~K}$ & $0.0005-0.002$ \\
\hline
\end{tabular}

Table 2 Comparison for 50 round trips (from (Oracle. 1995))

\begin{tabular}{lrr}
\hline Networks & Latency (secs) & Response time (secs) \\
\hline Wireless WAN & 4 & 200 \\
Modem & 0.3 & 15.0 \\
LAN & 0.002 & 0.1 \\
\hline
\end{tabular}

shows the dramatic discrepancies between bandwidth (kilobytes per second) and the network round trip times (latency) of the media.

Normally a client-server paradigm is utilized for most of the distributed computation. Considering a client-server application which exchanges approximately fifty messages, the Table. 2 shows how that application fares over a LAN, a phone line, and a wireless network.

The mobile computer thus has a highly constrained and fallible link and has limited battery storage capacity. Because of these reasons a mobile computer is more attuned to partially connected computing. The mobile agent paradigm provides this facility of partially connected computing and also the capability of performing functions inspite of intermittent connections. Thus mobile agent paradigm is suitable for the implementation of the MNMs. In order to enable the MNMs we are developing our own mobile agent environment called MAGENTA (Mobile AGENT for Administration). We also utilize the environment for decentralizing certain network management functions.

For implementing this scheme of MNMs, we model an indirect interaction. There is thus a concept of a static proxy in our design. One of the existing static managers is allocated to each portable computer to act as a proxy. The proxy acts as an intermediary between the portable computer and the server. The agent emanating from the portable computer on reaching the proxy conveys the requests to the proxy. The proxy tries to get the information from the server. In case the operation is successfully carried out, the agent before going back to the portable computer checks whether it is still connected. In case the proxy is not able to provide the information to the agent, it can either move to the server site or to the site of SNMP agents to obtain the 


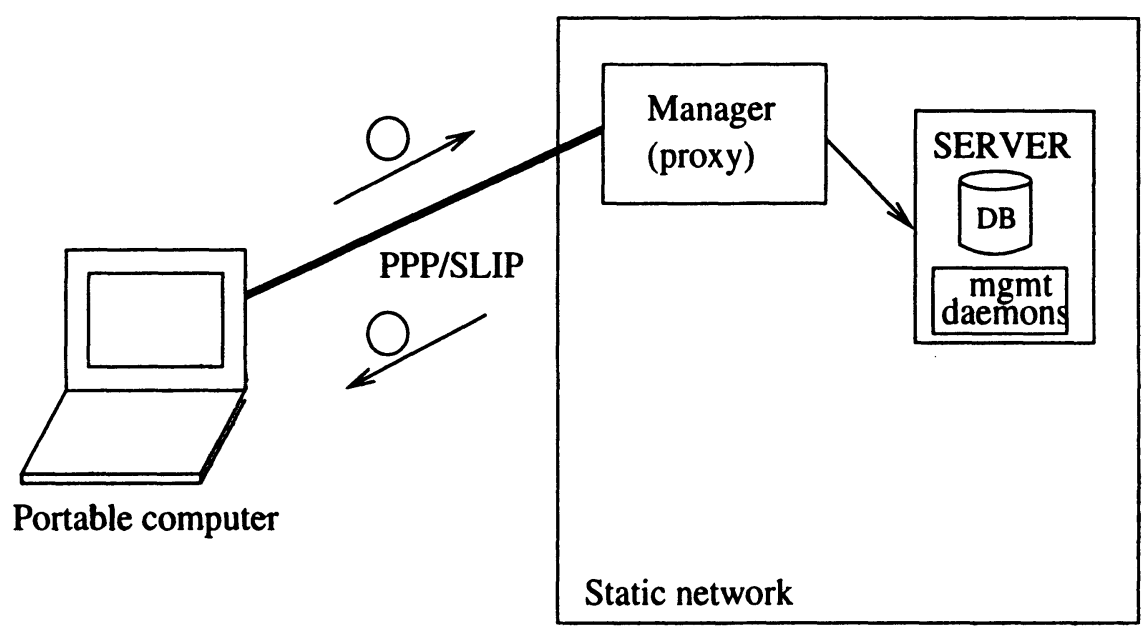

Figure 3 The MNM in tethered mode

necessary information. If the portable computer is connected it goes back to the portable computer otherwise the agent waits for the portable computer to connect again. The agents thus reduce the continuous usage of the highly constrained link and also take care of it fallibility. The tethered mode of operation is straightforward as depicted in Figure 3. In the tethered mode the portable is connected to the static network through a PPP/SLIP mechanism and the mobile manager runs on the portable which sends mobile agents to retrieve the required information from time to time. These agents collect the information and either return back immediately if the portable remains connected otherwise they wait and return back as soon as the portable is connected back.

The wireless mode of operation needs to be explained in detail. Normally in the area of wireless computing the total domain is divided into cells. Each of the cells have a Mobile Support Station (MSS) of their own. The responsibility of serving the Mobile Host (MH) as it moves from one cell to another cell changes from one MSS to another (Bakre, Badrinath. 1994)(Markku et al. 1995). The utilization of a static proxy serves us in good stead. The mobile agent as launched by the portable computer (MH) goes to the proxy which in turn obtains the required information. In the meanwhile if the $\mathrm{MH}$ moves to a new cell the new MSS is informed by the MH to retrieve the agent waiting at the proxy with the results. The proxy then delivers the agent at the new location of $\mathrm{MH}$ as shown in the Figure 4.

\section{MAGENTA ENVIRONMENT}

MAGENTA (Mobile AGENT for Administration) is our local mobile agent en- 


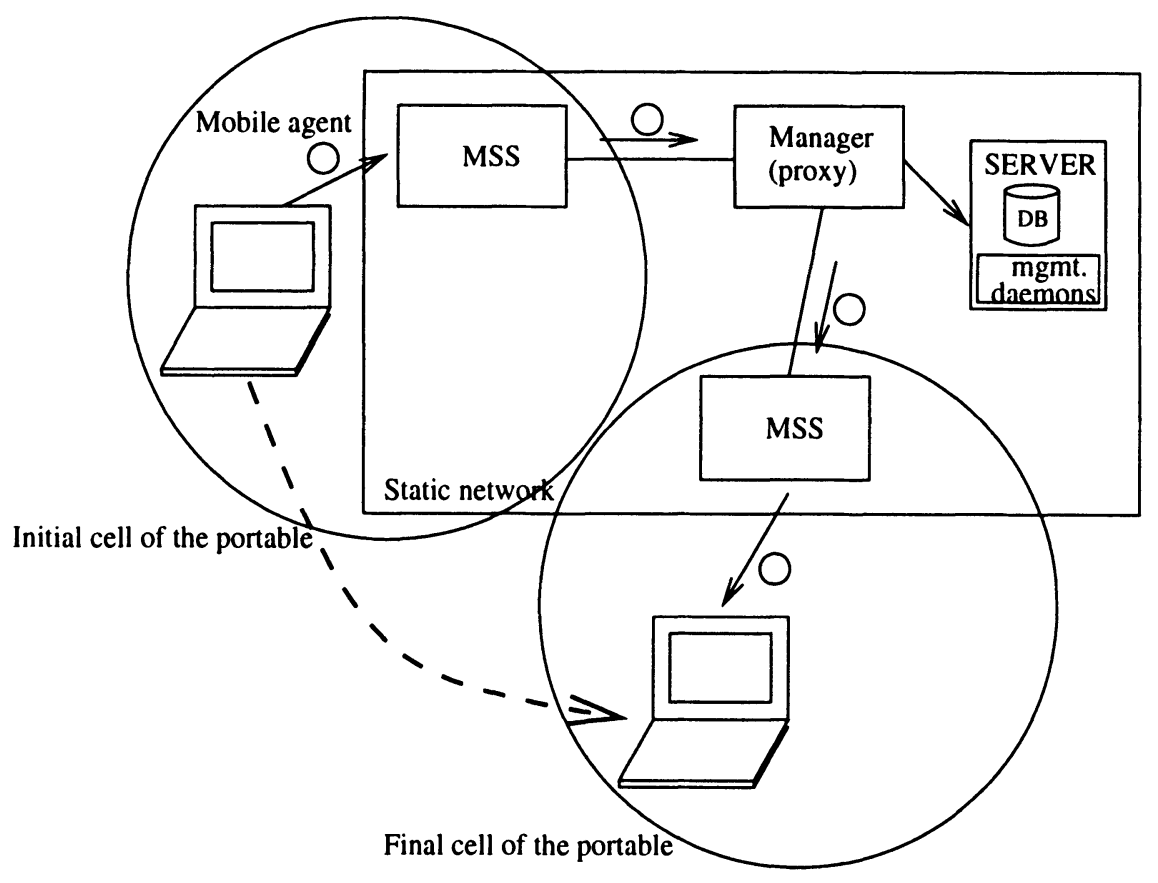

Figure 4 The MNM in wireless mode

vironment written in Java. The MAGENTA environment essentially comprises of the lieus and agents. The lieus are sites which provide the functionality of sending, receiving and executing the agents and of allowing the agents to access the local resources, if necessary as shown in Figure 5. Lieus can exist independently as in the case of network elements supporting SNMP agents and the network management server(s) but are integrated with the network managers on the network elements where the managers exist. Agents are mobile code which move from one lieu to another and perform a goal-directed behaviour. They exhibit the characteristics of autonomy, mobility, social ability, reactivity and proactiveness. The agents are autonomous as they exhibit control over their state and actions as they move from one lieu to another. They are reactive as they react to the changes in the environment and alter their behaviour accordingly. They are proactive because they take the initiative and have a predefined purpose. They are socially able as they can meet other agents.

In our environment mobile agents are autonomous programs and perform a predefined task and interact with the available resources in the optimum manner. An agent interacts with the resources that are available on the lieu where it executes its task. For security reasons, an access control is introduced: the agents are not given direct access to system resources and the access to any resource is always predefined and controllable by the lieu. Every agent carries 
a permit which identifies the agent and the origin of it. These permits determine the priority and the range of functionality of the agent. The MAGENTA environment provides autonomy to the agent by providing it the capability of operating without direct human intervention, they can utilize the move primitive to move to a destination without human intervention. The agents can change their itinerary by using a decide primitive. They exhibit social ability, interact with other agents and pass information amongst themselves through notes. They are reactive because they perceive their environment and respond to them in a timely fashion for example they have decide primitive to decide their itinerary depending on the availability of services at a lieu. They are proactive because they exhibit goal-directed behaviour by taking initiative. Each agent is provided with a purpose and they perform actions to fulfill the purpose.

In our case an agent can move from one lieu to another, thus occupying different lieus at different times, but only one lieu at time, i.e they cannot be fragmented. An agent moves to other destination by utilizing move instruction. This instruction can appear at arbitrary points, and once the instruction is called, the agent is transmitted to the destination machine with its state. The lieus handle all transmission details, including the possibility of the destination machine being disconnected.

In order to provide the knowledge of available services to the agent we utilize backward learning. An agent carries its own history, that is, the identifier of the sender that has launched it, the identifier of the lieus on which the agent has been and the services that the agent has used on each. So, when an agent arrives on a lieu, the lieu can read the agent's history and learns which are some services that the lieus, which the agent comes from, perform. Therefore, the lieu updates a list called other service list. This technique is utilized to update the already available information at the lieu given to them initially. If a lieu has no information about a service, the lieu gives the agent a list of lieus from which the agent can choose to go to and get the required information.

So, the logical state of the lieu in our environment comprises of:

- The list of available services "other service list".

- The list of it's own services, the "own service list" with all services that the lieu performs.

- The state of all the running agents.

- The list of all lieus which are up and working.

while the logical state of the agent comprises of:

- Purpose: The intention of the agent.

- Services Done: it is the agent's history. It can be null if the agent hasn't completed. This information is necessary for the backward learning. 


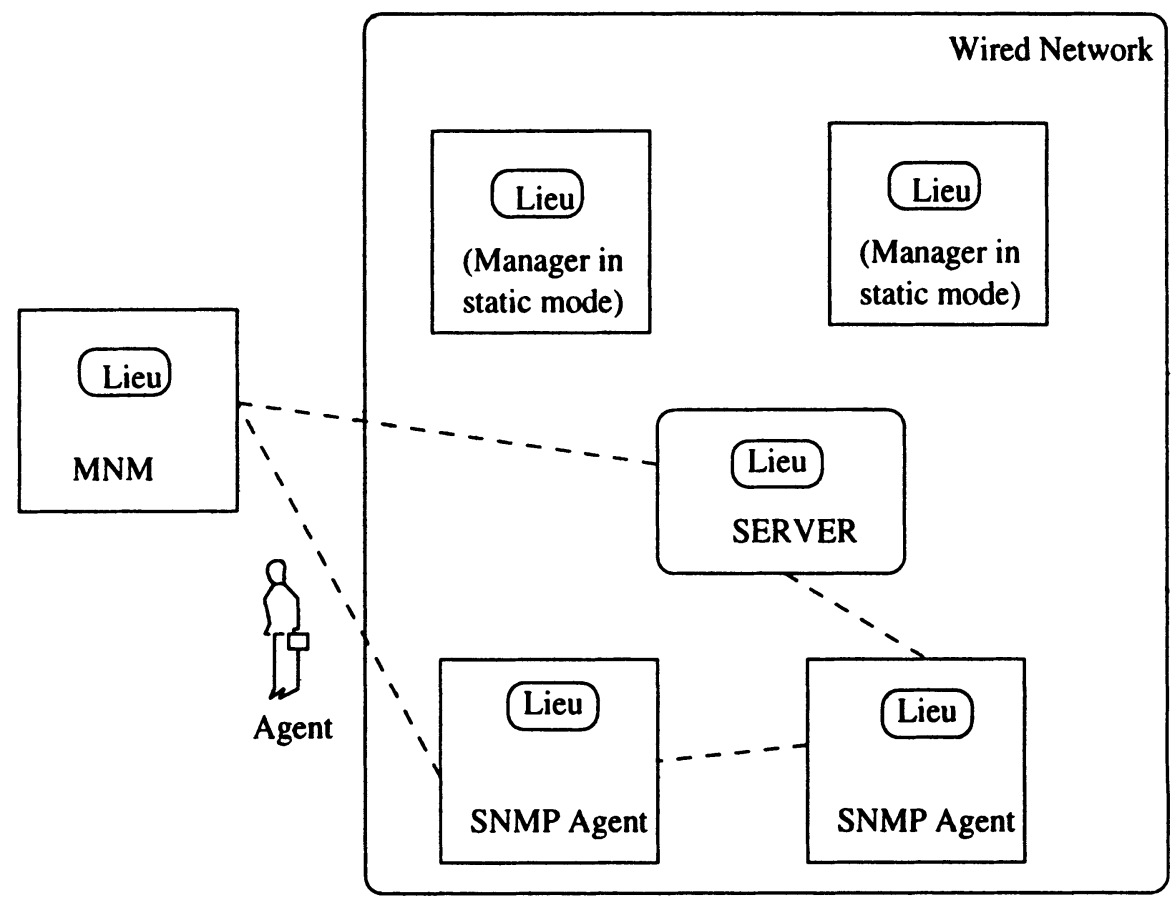

Figure 5 Utilization of mobile agents for network management in MAGENTA environment

- Next Service List: it can hold 0,1 , or more elements. The list carries the services that the agent hasn't accomplished yet.

- Sender: it is the address of the sender lieu which has launched the mobile agent. It is necessary to allow the agent to go back;

- Folder: In this the agent carries the results and responses.

- Permit: It is used to authenticate the sender lieu of the mobile agent, to control the resources and functions access.

- Note: In case there is a note to be passed to other agents.

The design of MAGENTA environment, thus is highly generic in nature and can be utilized for the administration of different services like data mining, electronic commerce and obviously network management.

\section{IMPLEMENTATION}

The Astrolog action as detailed above comprises of the managers and the servers. The implementation of manager and server is underway. A skeletal 
manager and server having limited functionality have been implemented. The server part comprises of a database, a communication mechanism, a network management system kernel which also comprises of a discovery daemon. The discovery daemon discovers the devices and populates the database. The NMS kernel also comprises of daemon which queries the SNMP agents executing on SNMP capable devices to know their status and stores the information in the database. The manager comprises of the GUI, the applications and a mechanism to access the information from the server. The managers operate in static or dynamic mode depending on their mode of communication which is either utilization of client-server mechanism or utilization of mobile agent technology respectively.

The implementation of MAGENTA environment is nearing completion. A small agent demonstrator has been implemented. The MAGENTA environment is written in Java and utilizes Java Object Serialization of JDK1.1.1. The agents are Java classes which migrate between the lieus which are integrated with the managers.

Java (Gosling, McGilton. 1995) was chosen for implementation of MNM and MAGENTA because it is architecturally neutral. It was intended to have multiple managers accessing the server from a variety of platforms, thus only the local managers needed to be platform-independent. It was a necessity in our case because Astrolab is a heterogeneous system. Java is also object-oriented and dynamically extensible and thus suitable for writing mobile agents. Java also provides the capability of native methods to access the local operating system and thus is ideally suited for obtaining system information. Because of these reasons Java has been recently used for network management applications like MIB browsing (AdventNet. 1997). Java also provides the capability of deriving meaningful meta-variables from the available SNMP variables to the local managers enabling them to monitor the local domains more effectively. The local managers are thus capable of providing the system users and the system administrator an easily portable network and system visualization and management platform. The managers connect to the management servers over the network through a high-level protocol or by utilizing mobile agents.

\section{CONCLUSION}

Astrolog contributes significantly to the utilization of mobile agents in the domain of network management. It introduces the unique concept of Mobile Network Manager (MNM) which would be extremely useful in the near future because of proliferation of wireless networks. It also furthers the cause of the utilization of mobile agents for resource constrained portable computers and as an essential mode of usage in the case of partially connected computing. It 
introduces the concept of a simple, portable, cost-effective, light-weight and mobile manager. It also introduces the generic MAGENTA environment for administration.

We intend to utilize MAGENTA environment for decentralizing most of the network management functions in the static mode of operation of the manager also. An elaborate study of the appropriateness of the mobile agent paradigm and the client-server paradigm for different network management functionalities is envisaged.

\section{REFERENCES}

AdventNet (1997) Advent NetMonitor Fabricated by Advent Network Management Inc., http://www.adventnet.com.

Bakre, A. and Badrinath, B.R. (1994) I-TCP: Indirect TCP for Mobile Hosts Technical Report DCS-TR-314, Department of Computer Science, Rutgers University, 1994.

Beale, R. and Wood, A. (1994) Agent Based Interaction.In People and Computers IX: Proceedings of HCI'94, Glasgow, UK, August 1994 239-245.

Case, J. Fedor, M. Schoffstall. M, and Davin, J. (1990) A Simple Network Management Protocol (SNMP) RFC 1157.

Colin, G. and Harrison et al. (1995) Mobile Agents: Are they a good idea? IBM T.J. Watson Research Center Technical Report, 1995.

CyberAgent (1997) Agent applications Fabricated by FTP Software, http://www.ftp.com/product/.

Goldzmith, G. and Yemini, Y. (1995) Decentralizing Control and Intelligence in Network Management. In the Proc. of 4th International Symposium on Integrated Network Management, Santa Barbara, CA, 1995.

Gosling, J. and McGilton, H. (1995) The Java Language Environment: A White paper Technical Report, Sun Microsystems, 1995.

Gray, R. Kotz, D. Nog, S. Rus,D. Cybenko, G. (1996) Mobile Agents for Mobile Computing Technical report PCS-TR96-285, Department of Computer Science, Dartmouth College, hanover, 1996.

Gray , R.S. (1995) Agent Tcl: Alpha Release 1.1, 1995. Available at http://www.cs.dartmouth.edu/ rgray/transportable.html.

Jennings, N. and Wooldridge, M. (1994) Software Agents. IEE Review, January 1994, 17-20.

Johansen, D. Renesse, R.V. and Schneider, F.B.(1995) An Introduction to TACOMA Distributed System Technical report CS-95-23, Department of Computer Science, Institute of Mathematical and Physical Sciences, University of Tromso., http://www.cs.uit.no/DOS/Tacoma/.

Maes, P. (1994) Agents that reduce work and information overload Communications of the ACM, 37(7):31-40, 1994 .

Magedanz, T. Rothermel, K. and Krause, S. (1996) Intelligent Agents: An Emerging technology for Next Generation Telecommunications INFO- 
COM 96, USA, March 24-28, 1996.

Kraus, S. (1997) MAGNA-A DPE-based Platform for Mobile Agents in Electronic Service Markets.Submission for ISADA '97-The Third International Symposium on Autonomous Decentralized Systems, 9-11 April 1997, Berlin, Germany.

Markku, Kojo. Alanko, Timo. Liljeberg, Mika. and Raatikainen, Kimmo. (1995) Enhanced Communication Services for Mobile TCP/IP Networking Technical Report C-1995-15, Deptt. of Computer Science, University of Helsinki.

Oracle. (1995) Oracle White Paper: Oracle Mobile Agents Technical Report, August, 1995.

Reilly, S. and Joseph, W.B. (1995) Natural negotiation for Believable Agents Technical report CMU-CS-95-164, School of Computer Science, Carnegie Mellon University, 1995.

Rose, M. and McCloghrie, K. (1990) Structure and Identification of Management Information for TCP/IP-based internets (SMI) RFC 1155.

Sahai, A. Billiart, S. and Morin, C. (1997(1)) Astrolog: A distributed and dynamic environment for network and system management.In the Proc. of 1st European Information Infrastructure User Conference, Stuttgart, Germany, Feb. 1997, http://www.irisa.fr/solidor/doc/pub97.html.

Sahai, A. Billiart, S. and Morin, C. (1997(2)) A portable and mobile manager for distributed system management.In the Proc. of Third Joint Conferenceon Information Sciences, Raleigh, USA, Mar. 1997, http://www.irisa.fr/solidor/doc/pub97.html.

Stallings. SNMP, SNMPv2 and CMIP: The practical guide to network management standards. Addison-Wesley publication, 1994.

Telscript (1996) Telescript at General Magic Inc. Information available at http://www.genmagic.com/Telescript/index.html.

Warrier, U. and Besaw, L. (1989) The Common Management Information Services and Protocols over TCP/IP (CMOT) RFC 1095. 